\title{
Phosphoinositide interacting regulator of TRP (Pirt) enhances TRPM8 channel activity in vitro via increasing channel conductance
}

\author{
Min TANG ${ }^{1,2}$, Guang-yi $\mathrm{WU}^{2}$, Xin-zhong DONG ${ }^{3}$, Zong-xiang TANG ${ }^{2, *}$ \\ ${ }^{1}$ College of Biology and Environmental Sciences, Jishou University, Jishou 416000, China; ${ }^{2}$ College of Basic Medicine, Nanjing \\ University of Chinese Medicine, Nanjing 210023, China; ${ }^{3}$ Howard Hughes Medical Institute, Johns Hopkins University School of \\ Medicine, Baltimore, MD 21205, USA
}

\begin{abstract}
Aim: Pirt is a two-transmembrane domain protein that regulates the function of a variety of ion channels. Our previous study indicated that Pirt acts as a positive endogenous regulator of the TRPM8 channel. The aim of this study was to investigate the mechanism underlying the regulation of TRPM8 channel by Pirt.

Methods: HEK293 cells were transfected with TRPM8+Pirt or TRPM8 alone. Menthol (1 mmol/L) was applied through perfusion to induce TRPM8-mediated voltage-dependent currents, which were recorded using a whole-cell recording technique. PIP2 (10 $\mu$ mol/L) was added into the electrode pipettes (PI was taken as a control). Additionally, cell-attached single-channel recordings were conducted in $\mathrm{CHO}$ cells transfected with TRPM8+Pirt or TRPM8 alone, and menthol ( $1 \mathrm{mmol} / \mathrm{L})$ was added into the pipette solution.

Results: Either co-transfection with Pirt or intracellular application of PIP2 (but not PI) significantly enhanced menthol-induced TRPM8 currents. Furthermore, Pirt and PIP2 synergistically modulated menthol-induced TRPM8 currents. Single-channel recordings revealed that co-transfection with Pirt significantly increased the single channel conductance.

Conclusion: Pirt and PIP2 synergistically enhance TRPM8 channel activity, and Pirt regulates TRPM8 channel activity by increasing the single channel conductance.
\end{abstract}

Keywords: TRPM8; menthol; Pirt; PIP2; PI; whole-cell recording; single channel conductance

Acta Pharmacologica Sinica (2016) 37: 98-104; doi: 10.1038/aps.2015.110; published online 14 Dec 2015

\section{Introduction}

TRPM8 was first identified as a $\mathrm{Ca}^{2+}$-permeable nonselective cation channel that can be activated by menthol and temperatures below $25^{\circ} \mathrm{C}^{[1,2]}$. In the peripheral nervous system (PNS), TRPM8 is expressed in the dorsal root ganglion (DRG) and trigeminal ganglia. The gating behavior of TRPM8 is regulated by several exogenous and endogenous factors. For example, the intracellular $\mathrm{pH}$ in the physiological range modulates the activation of TRPM8 by icilin and cold temperatures but not menthol ${ }^{[3]}$. The lysophospholipids and polyunsaturated fatty acids from phospholipase A2 (PLA2) mediate TRPM8 sensitization and activation ${ }^{[4]}$. Moreover, phosphatidylinositol4,5-bisphosphate $\left(\mathrm{PI}(4,5) \mathrm{P}_{2}\right.$ or PIP2), a common regulator of different ion channels including the transient receptor poten-

\footnotetext{
* To whom correspondence should be addressed.

E-mail zongxiangtang@njutcm.edu.cn

Received 2015-09-06 Accepted 2015-11-01
}

tial (TRP) channels ${ }^{[5-7]}$, participates in the activation of TRPM8 by the cold and menthol ${ }^{[8]}$.

Phosphoinositide interacting regulator of TRP (Pirt), a twotransmembrane domain protein specifically expressed in the PNS, was first identified as a positive modulator of TRPV1 via binding to PIP2 ${ }^{[9]}$. Pirt-null mice exhibit impaired pain behavior to capsaicin and noxious heat ${ }^{[9]}$. In addition, Pirt also contributes to both non-histamine- and histamine-dependent itch sensations ${ }^{[10]}$. Recently, we demonstrated that Pirt functions as an endogenous regulator of TRPM8 ${ }^{[11]}$. $\mathrm{Pirt}^{-/-}$mice displayed decreased behavioral responses to cool temperatures compared with $\mathrm{Pirt}^{+/+}$mice. TRPM8 channel currents that were induced by menthol and cool temperatures were significantly reduced in the Pirt/- DRG neurons. Pirt also inhibits purinergic receptor $\mathrm{P} 2 \mathrm{X} 3$ and reduces bladder overactivity ${ }^{[12]}$, but unlike the Pirt positive regulation of TRPV1, this inhibitory effect is mediated through the interaction between the Pirt N-terminal and $\mathrm{P} 2 \mathrm{X}^{[12]}$.

Pirt promotes TRPM8 channel activity ${ }^{[11]}$, but the underlying 
mechanism remains unclear. Here, we investigated the role of PIP2 and Pirt in the regulation of TRPM8 channel activity in a heterologous cell line. We demonstrated that both Pirt and PIP2 observably increased the voltage-dependent current of TRPM8 and its sensitivity to menthol. Moreover, Pirt and PIP2 displayed a synergistic regulatory effect on TRPM8. Lastly, Pirt enhanced the activity of the TRPM8 channel via increasing its conductance and change in open probability.

\section{Materials and methods Cell culture}

Human embryonic kidney (HEK) 293 cells were obtained from Dr Paul Worley's laboratory at Johns Hopkins University School of Medicine. The growth medium of HEK293 cells consisted of $90 \%$ DMEM, $10 \%$ fetal bovine serum, $50 \mathrm{U} / \mathrm{mL}$ penicillin, and $50 \mu \mathrm{g} / \mathrm{mL}$ streptomycin-glutamine (Invitrogen). The cells were cultured at $37^{\circ} \mathrm{C}$ in the presence of $95 \% \mathrm{O}_{2}$ and $5 \% \mathrm{CO}_{2}$. HEK293 cells were transfected with TRPM8+Pirt and TRPM8 alone mouse plasmids. An enhanced green fluorescent protein (GFP) cDNA tag was also used to confirm successfully transfected cells. Lipofectamine 2000 (Invitrogen) was used to deliver the cDNA. After $18-24 \mathrm{~h}$, the cells were plated onto glass coverslips coated with poly-D-lysine and laminin.

Chinese hamster ovary $(\mathrm{CHO})$ cells were cultured in a medium consisting of 90\% DMEM, 10\% FBS, $100 \mathrm{U} / \mathrm{mL}$ penicillin, and $100 \mu \mathrm{g} / \mathrm{mL}$ streptomycin. GlutaMAX (Gibco 35050061) was also added. The $\mathrm{CHO}$ cells were cultured using the same methods as for the HEK cells. The single-channel recording experiments were performed after an additional 1-2 d.

\section{Electrophysiological recording}

Whole-cell recordings were performed in HEK293 cells transfected with TRPM8+Pirt+GFP and TRPM8+GFP. The transfected cells were identified using GFP fluorescence and were selected for recording. To diminish the effect of drugs used in the previous cell recording, only one cell per coverslip was tested. The current signal was collected and measured with an Axon 700B amplifier and the pClamp9.2 software package (Axon Instrument), respectively. The electrodes were pulled (Sutter, model P-97) from borosilicate glass (Sutter Inc) and had a resistance of 2-4 M $\Omega$. The series resistance was usually less than $10 \mathrm{M} \Omega$ and was not compensated. All experiments were performed at room temperature $\left(\sim 25^{\circ} \mathrm{C}\right)$. The electrophysiological recordings were performed by one individual who was blinded to the genotype. After breaking the cell membrane and forming a stable whole-cell recording configuration, the capacitance values were obtained from the amplifier.

For whole-cell voltage-clamp recordings, the train of voltage steps ranged from $-100 \mathrm{mV}$ to $+100 \mathrm{mV}$ with $20 \mathrm{mV}$ step sizes that lasted $20 \mathrm{~ms}$. To confirm the specificity of the TRPM8 channel to the stimulation of the voltage changes, a train of voltage step stimulations were provided at the peak with 1 $\mathrm{mmol} / \mathrm{L}$ menthol inducing an inward current. The holding potential was kept at $-60 \mathrm{mV}$ when various voltage stimuli were applied to the recorded cells. Whole-cell recording data were sampled at $2 \mathrm{kHz}$ and filtered at $0.5 \mathrm{kHz}$ for analysis.

For singe-channel recording, the cell-attached recording mode was adapted in CHO cells expressing TRPM8+Pirt and TRPM 8 alone. Menthol $(1 \mathrm{mmol} / \mathrm{L})$ was added into the electrode pipette to detect TRPM8 channel activity, but it was absent from the bath solution. Voltage steps ranging from $-80 \mathrm{mV}$ to $+80 \mathrm{mV}$ with a $20 \mathrm{mV}$ step size were performed to analyze TRPM8 single-channel activity. The single-channel currents were sampled at $0.5 \mathrm{kHz}$ and filtered with an 8-pole with a low pass Bessel filter at $0.1 \mathrm{kHz}$. As the dwelling time of TPRM8 single-currents was $>0.5 \mathrm{~s}$, the single-channel unitary current (i) was determined from the best-fit Gaussian distribution of amplitude histograms. Single-channel activity was calculated from $\mathrm{NPo}=\mathrm{I} / \mathrm{i}$, where I is the mean total current in a patch, and $i$ is the unitary current at this voltage. The open probability (Po) for the main conductance is presented in the figures. For single-channel slope conductance, linear fitting was used separately for the positive and negative holding potentials. The single-channel recording data were analyzed with Clampfit 9.2 software. The "event detection" function option in the main menu was used to analyze the single channel current traces, determine the number of channels in the patch $(\mathrm{N})$, and calculate the conductance.

\section{Drugs and solutions}

For the whole-cell recordings, HEK293 cells were perfused with an extracellular solution comprising $140 \mathrm{mmol} / \mathrm{L} \mathrm{NaCl}, 4$ $\mathrm{mmol} / \mathrm{L} \mathrm{KCl}, 2 \mathrm{mmol} / \mathrm{L} \mathrm{CaCl}_{2}, 2 \mathrm{mmol} / \mathrm{L} \mathrm{MgCl}_{2}, 10 \mathrm{mmol} / \mathrm{L}$ HEPES, and $5 \mathrm{mmol} / \mathrm{L}$ glucose, and the $\mathrm{pH}$ was adjusted to 7.38 using $\mathrm{NaOH}$. The intracellular pipette solution comprised $135 \mathrm{mmol} / \mathrm{L} \mathrm{KCl}, 3 \mathrm{mmol} / \mathrm{L} \mathrm{MgATP}, 0.5 \mathrm{mmol} / \mathrm{L}$ $\mathrm{Na}_{2} \mathrm{ATP}, 1.1 \mathrm{mmol} / \mathrm{L} \mathrm{CaCl}_{2}, 2 \mathrm{mmol} / \mathrm{L}$ EGTA, and $5 \mathrm{mmol} / \mathrm{L}$ glucose. The $\mathrm{pH}$ was adjusted to 7.38 using $\mathrm{KOH}$, and the osmolarity was adjusted to $300 \mathrm{mOsm}$ using sucrose. For the PIP2 and PI experiments, $10 \mu \mathrm{mol} / \mathrm{L}$ PIP2-diC8 or PI-diC 8 was added to the intracellular pipette solution. Menthol was used as a TRPM8 agonist. It was dissolved in ethanol $(100 \mathrm{mg} / \mathrm{mL})$, stored at $-20^{\circ} \mathrm{C}$, and diluted with extracellular solution to different concentrations. All of the solutions were applied to the cells at a rate of $1 \mathrm{~mL} / \mathrm{min}$ via a gravity-fed perfusion system (VC-6 Six Channel Valve Controller, Warner Instruments)

For the single-channel recordings, the bath solution for recording $\mathrm{CHO}$ cells comprised $100 \mathrm{mmol} / \mathrm{L} \mathrm{K}$-gluconate, 4 $\mathrm{mmol} / \mathrm{L} \mathrm{KCl}, 1 \mathrm{mmol} / \mathrm{L} \mathrm{MgCl}_{2}, 1 \mathrm{mmol} / \mathrm{L}$ EGTA, $10 \mathrm{mmol} / \mathrm{L}$ glucose, and $10 \mathrm{mmol} / \mathrm{L}$ HEPES (pH 7.3). The pipette solution for the single-channel recordings included $100 \mathrm{mmol} / \mathrm{L}$ Na-gluconate, $10 \mathrm{mmol} / \mathrm{L} \mathrm{NaCl}, 1 \mathrm{mmol} / \mathrm{L} \mathrm{MgCl}_{2}, 2 \mathrm{mmol} / \mathrm{L}$ $\mathrm{CaCl}_{2}, 10 \mathrm{mmol} / \mathrm{L}$ glucose, and $10 \mathrm{mmol} / \mathrm{L}$ HEPES (pH 7.3).

\section{Data analysis}

The electrophysiological data were analyzed and fitted using Clampfit (Axon instruments, Foster city, CA, USA) and Origin Pro 8 (Origin Lab, USA) software. All of the data were analyzed with an unpaired Student's $t$-test and expressed as the mean \pm standard errors of the means (SEM). The statistical significance was set at $P<0.05$. 


\section{Results}

Pirt enhances TRPM8-mediated voltage-dependent currents via PIP2

We previously demonstrated that Pirt increased TRPM8mediated currents via positively charged amino acids residues in its $C$ terminal ${ }^{[11]}$. To investigate the effect of PIP2 on Pirtmediated regulation of TRPM8, we increased the intracellular PIP2 concentration by adding $10 \mu \mathrm{mol} / \mathrm{L}$ of PIP2 into the electrode pipette. A $10 \mu \mathrm{mol} / \mathrm{L}$ PI solution was used as a control. We measured the current-voltage relationships in HEK293 cells expressing TRPM8+Pirt and TRPM8 alone. In the recordings, the depolarizing voltage steps ranging from $-100 \mathrm{mV}$ to $+100 \mathrm{mV}$ were applied to induce currents in the presence of $1 \mathrm{mmol} / \mathrm{L}$ menthol. The background current was examined prior to the addition of menthol, and an identical protocol was performed when the inward current reached the maximum menthol perfusion, which indicated the maximum opening state of the TRPM8 channel (Figure 1A and 1D). The subtrac- tion of two currents that were evoked by the background current (before) and the menthol inward currents (after) should identify a relatively pure TRPM8-mediated voltage-dependent current (Figure 1B and 1E). We observed that the current density was significantly larger in TRPM8+Pirt cells than in TRPM8 cells at potentials ranging from $-100 \mathrm{mV}$ to $+100 \mathrm{mV}$ (Figure 1C). In cells transfected with TRPM8+Pirt, the current density was significantly greater with the addition of PIP2 than with the addition of PI (Figure 1F). Taken together, these results indicate that Pirt may enhance TRPM8-mediated voltage-dependent currents via PIP2.

Pirt and PIP2 contribute synergistically to the TRPM8-mediated voltage-dependent current

As we previously demonstrated that Pirt could bind PIP2 and form a complex with TRPM ${ }^{[9]}$, we hypothesized that Pirt and PIP2 contributed synergistically to TRPM8-mediated voltagedependent activity. We observed that Pirt-overexpressing
A

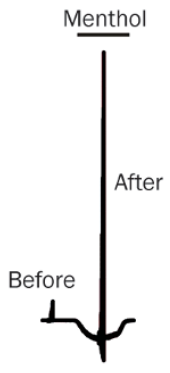

D

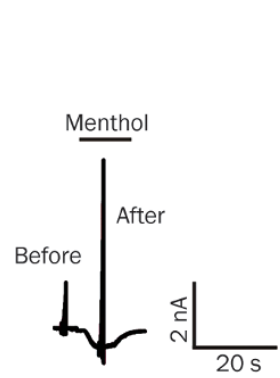

B

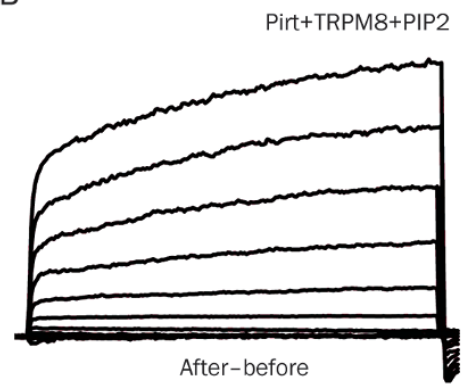

E

TRPM8+PIP2

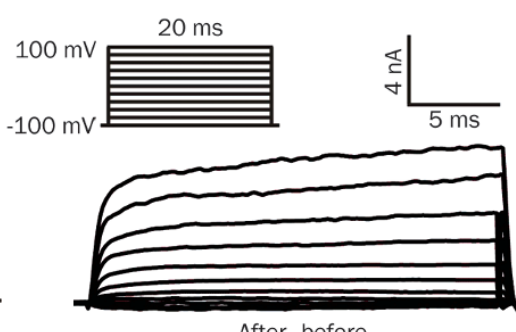

C $\rightarrow$ - Pirt+TRPM8+PIP2

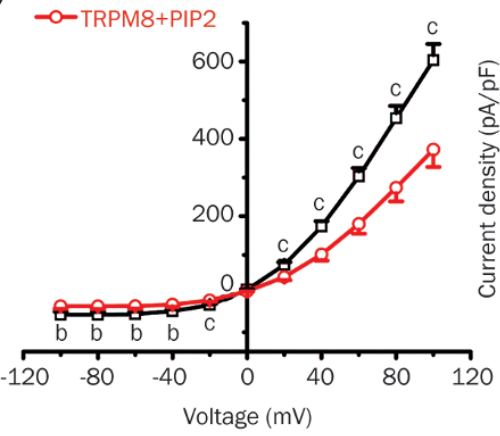

F

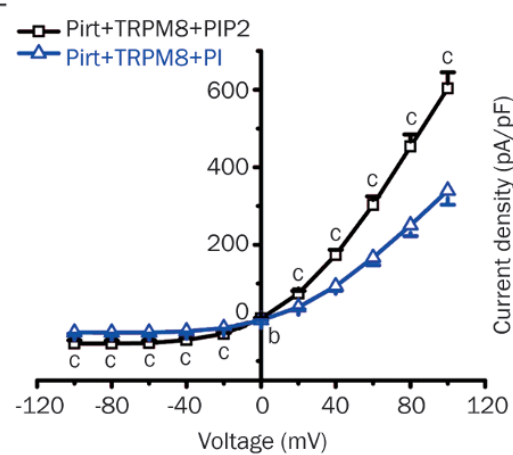

Figure 1. Pirt increases the TRPM8-mediated voltage-dependent currents via PIP2. (A) Representative current traces from TRPM8 stably expressed in HEK293 cells co-transfected with Pirt (Pirt+TRPM8+PIP2, $n=21)$, where PIP2 (10 $\mu \mathrm{mol} / \mathrm{L})$ was added into the electrode pipette. Whole-cell inward currents evoked by $1 \mathrm{mmol} / \mathrm{L}$ menthol were analyzed using a series of 20-ms step pulses ( $-100 \mathrm{mV}$ to $+100 \mathrm{mV}$ in $20 \mathrm{mV}$ steps from $-60 \mathrm{mV}$ holding potential; the protocol is shown in Figure 1E). Before, background currents; after, currents elicited during menthol perfusion. (B) The net currents mediated by TRPM8 were calculated from (A). (C) I-V curves constructed from TRPM8-mediated net currents (after-before) for HEK293 cells stably expressing TPRM8 alone (TRPM8+PIP2, $n=21$, red line) or co-transfected with Pirt (Pirt+TRPM8+PIP2, $n=21$, black line). PIP2 (10 $\mu$ mol/L) was added into the electrode pipette. (D) Menthol (1 mmol/L)-evoked inward current responses of HEK293 cells stably expressing TRPM8 alone (TRPM8+PIP2, $n=21$ ) were analyzed with the same protocol described in $(A)$, where PIP2 $(10 \mu \mathrm{mol} / \mathrm{L})$ was added into the electrode pipette. (E) The net currents mediated by TRPM8 were calculated from (D). (F) I-V curves constructed from TRPM8-mediated net currents (after-before) for HEK293 cells stably

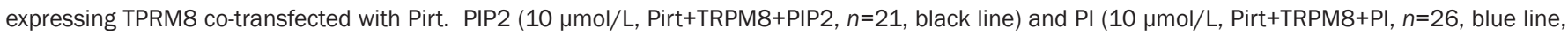
the representative current traces are not shown) were added into the electrode pipette. The reversal potentials for groups (C and $\mathrm{F}$ ) of cells were close to $0 \mathrm{mV}$, characteristic of a TRPM8-mediated current. Each value is presented as the mean \pm SEM. ${ }^{b} P<0.05,{ }^{\mathrm{C}} P<0.01$, unpaired $t$-test. 
A

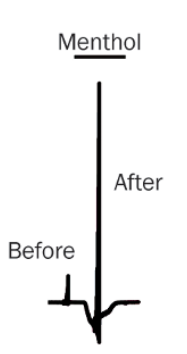

D

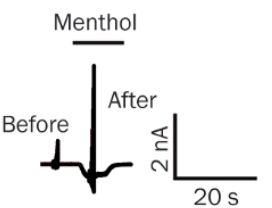

B

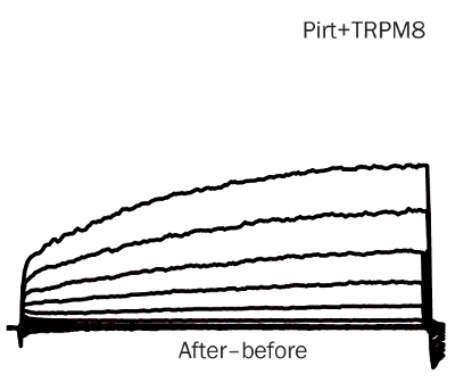

E

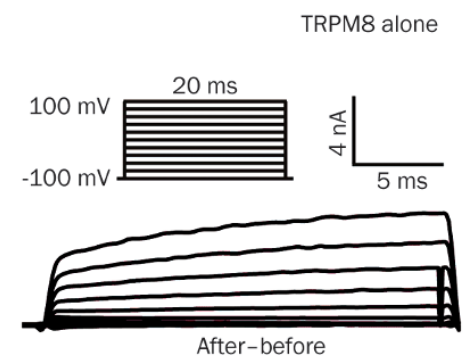

C $\rightarrow$-Pirt+TRPM8+PIP2

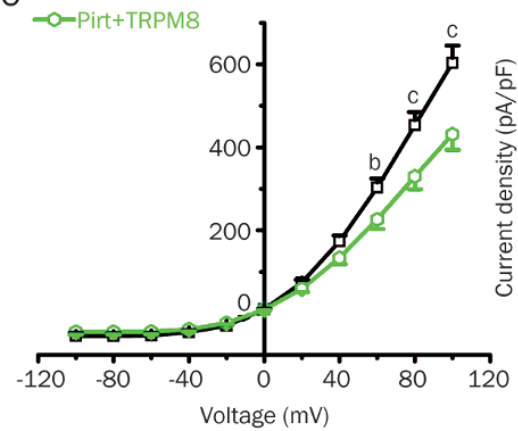

$\mathrm{F}$

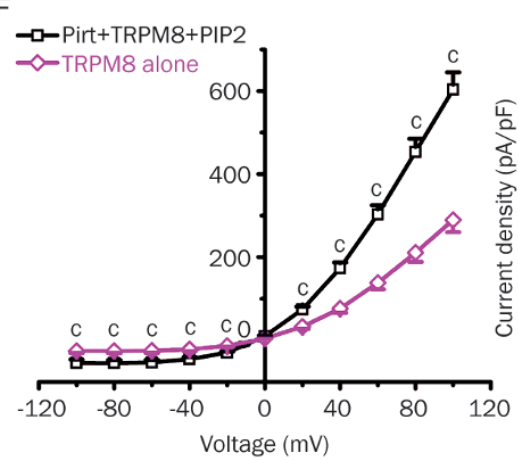

Figure 2. Pirt and PIP2 both modulate the TRPM8-mediated voltage-dependent current. (A) Representative current traces from HEK293 cells stably expressing TRPM8 co-transfected with Pirt (Pirt+TRPM8, $n=29$ ). (B) The net currents mediated by TRPM8 were calculated from (A). (C) I-V curves constructed from TRPM8-mediated net currents (after-before) for HEK293 cells stably expressing TPRM8 co-transfected with Pirt. PIP2 was added into the electrode pipette (Pirt+TRPM8+PIP2, $n=21$, black line) or not (Pirt+TRPM8, $n=29$, green line). (D) Menthol (1 mmol/L)-evoked inward current responses of HEK293 cells stably expressing TRPM8 alone (TRPM8 alone, $n=28$ ). (E) The net currents mediated by TRPM8 were calculated from (D). (F) I-V curves constructed from TRPM8-mediated net currents (after-before) for HEK293 cells stably expressing TPRM8 between Pirt+TRPM8+PIP2 ( $n=21$, black line) and TRPM8 alone ( $n=28$, purple line). Similar to Figure 1, The reversal potentials for groups (C and F) of cells were close to $0 \mathrm{mV}$. Each value is presented as the mean \pm SEM. ${ }^{b} P<0.05$, ${ }^{C} P<0.01$, unpaired $t$-test.

cells exhibited a significantly higher TRPM8-mediated voltagedependent current density in the presence of PIP2 compared to the PI control (Figure 2A, 2B and 2C). Furthermore, compared with TRPM8 alone, Pirt+TRPM8+PIP2 exhibited a significantly larger current density in the whole range of holding voltage potentials except at $0 \mathrm{mV}$, which indicates the reversal potential of most TRP channels (Figure 2D, 2E and 2F). Taken together, these data suggest that Pirt and PIP2 contribute synergistically to modulate the TRPM8-mediated voltage-dependent current.

PIP2 but not PI participates in the regulation of Pirt-mediated TRPM8

As we observed that Pirt and PIP2 enhanced the excitability of the TRPM8 channel in a voltage-dependent manner, we speculated that Pirt and PIP2 could form a complex that regulated TRPM8 function. We measured the TRPM8-mediated inward current in the presence of $1 \mathrm{mmol} / \mathrm{L}$ menthol applied to the bath solution. In this test, all recording cells were held at -60 $\mathrm{mV}$ at voltage-clamp. Similar to the previous test, PI was added into the electrode pipette solution during the recording process, and the same stimulus protocol was adopted.
The current density did not significantly differ between TRPM8+Pirt and TRPM8 alone after PI was added into the electrode pipette solution (Figure 3B, 3D and 3E). Similarly, in the TRPM8 alone overexpression cells, PIP2 and PI did not cause observable differences in the inward current (Figure 3E). In addition, we measured the capacitance of all recorded cells and observed no significant difference among these groups (Figure 3F). However, the inward current induced by menthol was significantly larger in the presence of PIP2 than in the presence of PI (Figure 3A, 3B and 3E). When the intracellular solution included PIP2, the inward current was significantly larger in the cells overexpressing TRPM8+Pirt than in those overexpressing TRPM8 alone (Figure 3A, 3C and 3E). Thus, these data suggest that Pirt enhances TRPM8 activity in response to menthol via PIP2 but not PI.

\section{Pirt increases the conductance of TRPM8 at a single channel level}

We previously demonstrated that Pirt increased the TRPM8 channel activity in response to electrical and chemical stimuli, but it did not affect the expression level of the TRPM8 protein $^{[11]}$. Therefore, we hypothesized that Pirt might modulate 
A

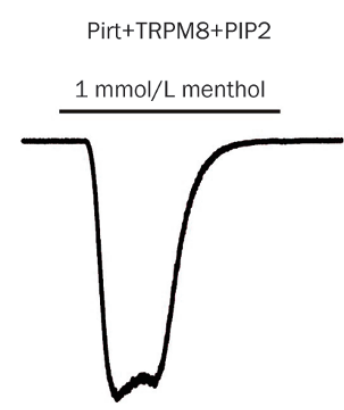

C

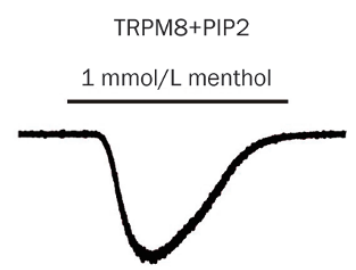

B

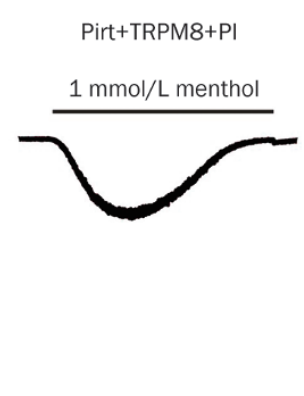

D

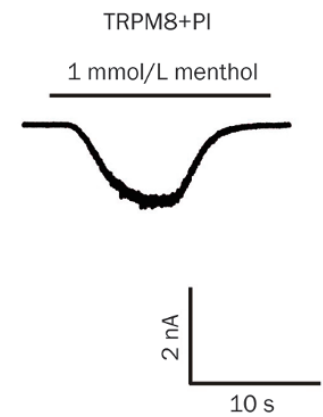

E

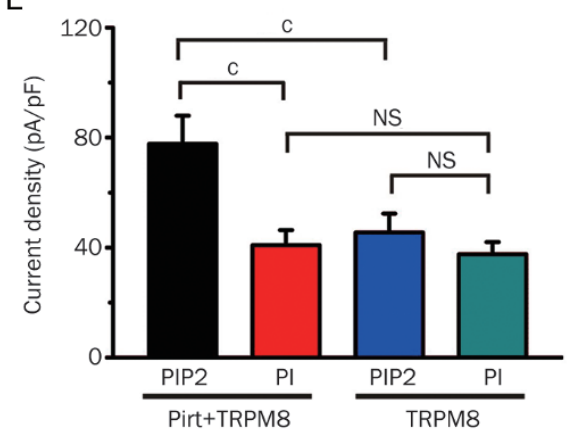

F

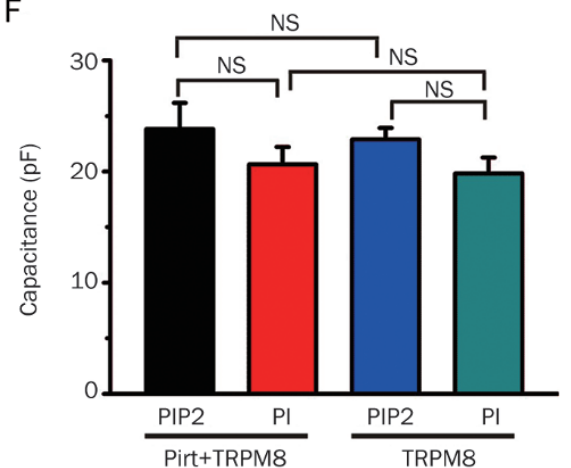

Figure 3. Pirt enhances TRPM 8 sensitivity in response to menthol via PIP2 but not PI. (A) Representative inward current response to $1 \mathrm{mmol} / \mathrm{L}$ menthol in HEK293 cells stably expressing TRPM8 co-transfected with Pirt, where PIP2 (10 $\mu \mathrm{mol} / \mathrm{L})$ was added into the electrode pipette (Pirt+TRPM8+PIP2, $n=12)$. (B) Representative inward current response to $1 \mathrm{mmol} / \mathrm{L}$ menthol in HEK293 cells stably expressing TRPM 8 co-transfected with Pirt, where PI $(10 \mu \mathrm{mol} / \mathrm{L}$ ) was added into the electrode pipette (Pirt+TRPM8+PI, $n=17$ ). (C) Representative inward current response to $1 \mathrm{mmol} / \mathrm{L}$ menthol in $\mathrm{HEK} 293$ cells stably expressing TRPM8 without Pirt, where PIP2 $(10 \mu \mathrm{mol} / \mathrm{L})$ was added into electrode pipette (TRPM8+PIP2, $n=13)$. (D) Representative inward current response to $1 \mathrm{mmol} / \mathrm{L}$ menthol in TRPM8 stably transfected HEK293 cells without Pirt, where the PI (10 $\mu \mathrm{mol} / \mathrm{L})$ was added into the electrode pipette (TRPM8+PI, $n=12$ ). (E) The current density among groups (A, B, C, and D) calculated from the current amplitude normalized to the cell capacitance was statistically compared. (F) The cell capacitance for four groups (A, B, C, and D) was statistically compared. The value is presented as the mean \pm SEM. ${ }^{\circ} P<0.01$, unpaired $t$-test. NS, non-significant difference.

the gating properties of the TRPM8 channel. To test this hypothesis, we performed cell-attached single channel recordings in $\mathrm{CHO}$ cells overexpressing TRPM8+Pirt and TRPM8 alone. As expected, when $1 \mathrm{mmol} / \mathrm{L}$ of menthol was added to the patch electrodes solution, various unitary currents were observed in both the cells overexpressing TRPM8+Pirt and TRPM8 alone at various holding potentials (Figure 4A and $4 \mathrm{~B})$. In contrast to TRPM8 alone cells, the unitary current was robustly enhanced in cells overexpressing TRPM8+Pirt at -60 $\mathrm{mV}$ and $+60 \mathrm{mV}$ (Figure $4 \mathrm{~A}$ and $4 \mathrm{~B}$ ). At negative holding voltage ranges, the channel conductance was significantly larger in TRPM8+Pirt cells than in TRPM8 alone cells at -80 and -60 $\mathrm{mV}$ holding potentials (Figure $4 \mathrm{C}$ ). The conductance was also significantly larger in TRPM8+Pirt cells than in TRPM8 alone cells at $+40 \mathrm{mV}$ to $+80 \mathrm{mV}$ holding potentials (Figure 4D). These data indicate that Pirt increases TRPM8 single channel conductance. In regard to the open probability of the channel, we did not observe significant differences between TRPM8+Pirt cells and TRPM8 alone cells at most holding potentials except at $+60 \mathrm{mV}$ (Figure $4 \mathrm{E}$ ). The number of open channels in the TRPM8+Pirt group and the TRPM8 alone group were plotted as Gaussian distributions. Pirt observably shifted the fitted curve to the right (Figure 4F). Taken together, these data suggest that Pirt increases the activity of TRPM8 by changing the conductance of the channel.

\section{Discussion}

PIP2 has been shown to modulate the physiological function of a variety of ion channels by acting as the substrate for cleavage by the enzyme phospholipase C (PLC ${ }^{[5,6]}$. PIP2 has been proposed to underlie the regulation of inwardly rectifying $\mathrm{K}^{+}$ (Kir) channels by diverse factors such as $\mathrm{G}$ protein, sodium, and magnesium ions, phosphorylation, and $\mathrm{pH}^{[13-16]}$. PIP2 also regulates various TRP channels by numerous exogenous and endogenous ligands and physical stimuli ${ }^{[17-24]}$. We previously demonstrated that Pirt directly enhanced the activity of the TRPM8 channel as an endogenous regulatory factor ${ }^{[11]}$. In the present study, we observed that PIP2 robustly increased the Pirt's regulatory function of TRPM8. Although we know that PIP2 and Pirt could interact with each other, it was not clear how a PIP2-Pirt enhanced the activity of TRPM8. PIP2 and Pirt may form a complex that binds to the TRP domain of 
A
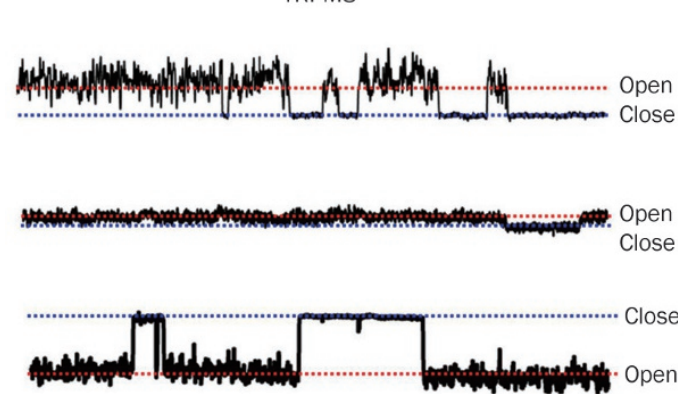

B
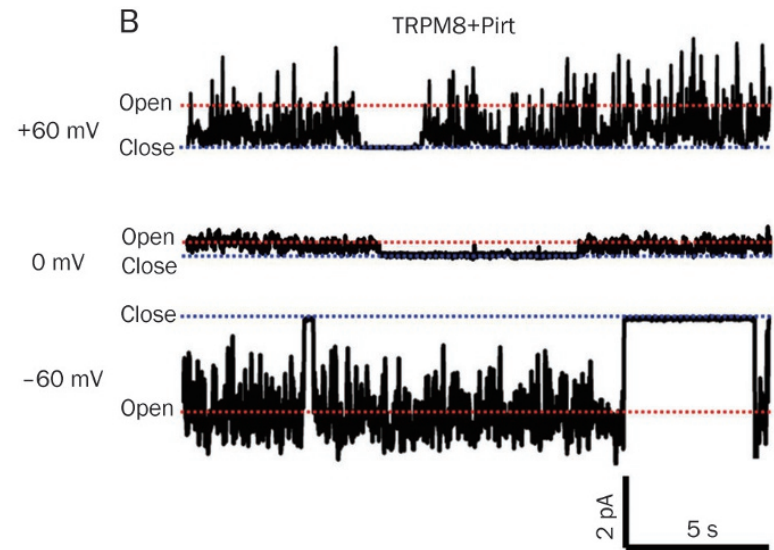

D

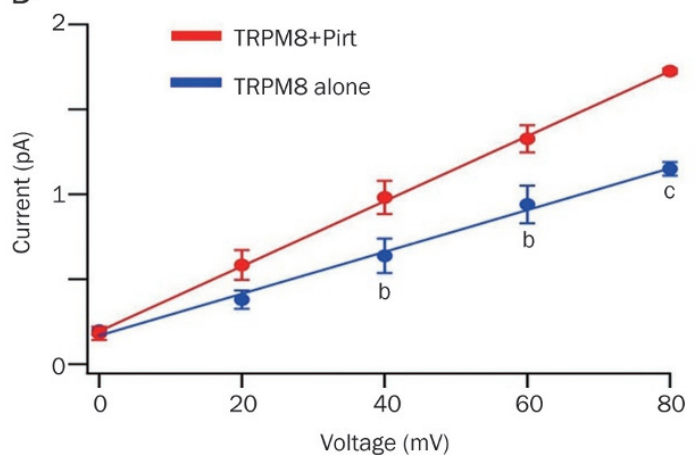

C

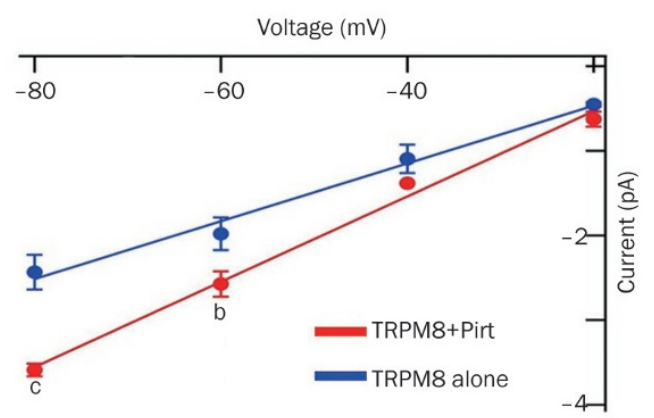

E

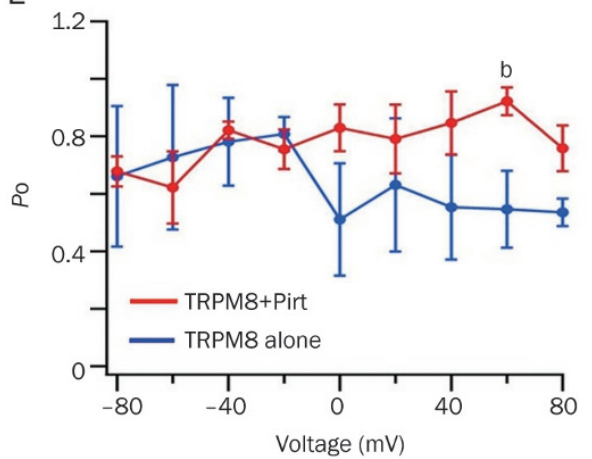

F

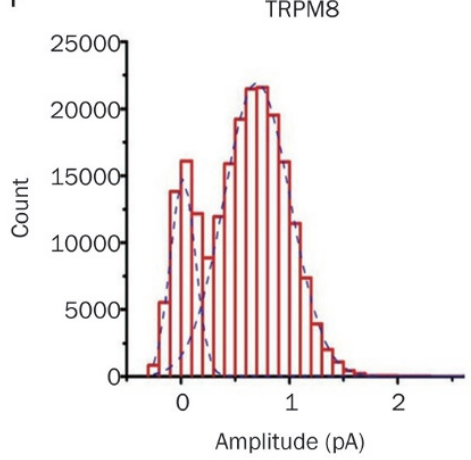

TRPM8+Pirt

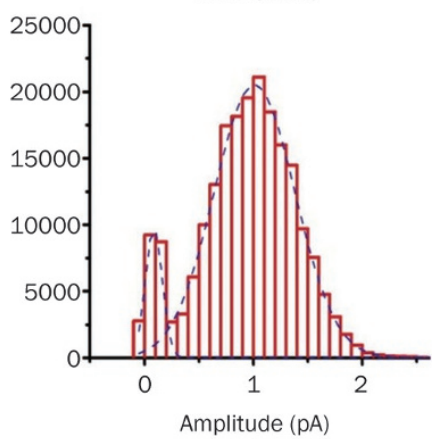

Figure 4. The unitary current trace of TPRM 8 in transfected $\mathrm{CHO}$ cells at different holding potentials. (A) Representative single-channel trace of $\mathrm{CHO}$ cells stably expressing TRPM8 without Pirt at voltages ranging from $+60 \mathrm{mV}$ to $-60 \mathrm{mV}(n=5)$. Red and blue dashed lines represent the open and closed conditions of TRPM8, respectively. (B) Representative unitary trace of TRPM8 with Pirt $(n=5)$. (C) At negative holding potential ranges from $-80 \mathrm{mV}$ to $-20 \mathrm{mV}$, single channel current-voltage plots of TRPM8 without Pirt (blue line) and TRPM8 with Pirt (red line). The data are fitted to a linear function with a slope of $37.31 \pm 1.2 \mathrm{ps}$ (TRPM8 without Pirt, $n=5$ ) and $50.36 \pm 1.95$ ps (TRPM8 with Pirt, $n=5$ ). (D) At positive holding potential ranges from $+20 \mathrm{mV}$ to $+80 \mathrm{mV}$, single channel current-voltage plots of TRPM 8 without Pirt (blue line, $n=5$ ) and TRPM 8 with Pirt (red line, $n=5$ ). (E) The open probability of the TRPM 8 channel co-transfected with Pirt (red line, $n=5$ ) or not (blue line, $n=5$ ) at different holding potentials. ( $F$ ) All-point histogram of the TRPM 8 channel co-transfected with Pirt (right, $n=5$ ) or without (left, $n=5$ ). The mean current amplitude at each point is obtained through the allpoint histogram and fit with a Gaussian distribution. Each value is presented as the mean \pm SEM. ${ }^{b} P<0.01,{ }^{c} P<0.01$, unpaired $t$-test.

TRPM8 channel to regulate the function of TRPM8 by changing the properties of the gated channel.

We previously determined that Pirt positively regulates the activity of TRPM8 but does not change the expression level of the TRPM8 protein ${ }^{[11]}$. Therefore, we propose that Pirt improves the voltage-dependent activity of TRPM8 upon menthol stimulation by changing the gating properties of the
TRPM8 channel. In our cell-attached single channel recording test, we obtained single-channel data from $\mathrm{CHO}$ cells overexpressing TRPM8+Pirt and TRPM8 alone and analyzed the gating properties of TRPM8. We observed that the conductance of the TRPM8 channel was significantly augmented in cells overexpressing TRPM8+Pirt compared with cells overexpressing TRPM8 alone (Figure 4C and 4D), indicating 
that the ion channel pore size was enlarged in the presence of a Pirt-TRPM8 interaction. However, the open probability of the TRPM8 channel did not change observably at different pipette potentials from $-80 \mathrm{mV}$ to $+80 \mathrm{mV}$, except at $+60 \mathrm{mV}$ (Figure 4E). In addition, the Gauss distribution of the channel open probability was also shifted to the right in TRPM8+Pirt cells (Figure $4 \mathrm{~F}$ ), indicating that more channels were not fully opened in TRPM8+Pirt cells than in TRPM8 alone cells. These multiple sub-conductance characteristics likely cause the larger current observed in TRPM8+Pirt overexpressing cells. Therefore, Pirt enhances the current of the TRPM8 channel mainly through raising the conductance.

In summary, PIP2 and Pirt together robustly increased the excitability of the TRPM8 channel likely through forming a complex. Moreover, Pirt enhanced the current of TRPM8 mainly through improving the conductance of the channel.

\section{Acknowledgements}

This work was supported by the National Natural Science Foundation of China to Zong-xiang TANG (31271181 and 31471007), by Oversea, Hong Kong \& Macao Scholars Collaborated Researching Fund to Xin-zhong DONG and Zong-xiang TANG (31328012), from the Project Funded by the Priority Academic Program Development of Jiangsu Higher Education Institutions (PAPD), sponsored by "Qing Lan Project" in Jiangsu Province and from the Jiangsu Collaborative Innovation Center of Traditional Chinese Medicine (TCM) Prevention and Treatment of Tumor, Nanjing University of Chinese Medicine, China. This work was also supported by Cooperative Innovation Center for Molecular Target New Drug Study, Research and Innovation Project of Hunan Province (CX2015B553) to Min TANG.

\section{Author contribution}

Min TANG performed the experiment and wrote the manuscript; Guang-yi WU helped to perform the electrophysiological experiment; Xin-zhong DONG and Zong-xiang TANG designed the study; Zong-xiang TANG wrote the final manuscript.

\section{References}

1 McKemy DD, Neuhausser WM, Julius D. Identification of a cold receptor reveals a general role for TRP channels in thermosensation. Nature 2002; 416: 52-8.

2 Peier AM, Moqrich A, Hergarden AC, Reeve AJ, Andersson DA, Story $\mathrm{GM}$, et al. A TRP channel that senses cold stimuli and menthol. Cell 2002; 108: 705-15.

3 Andersson DA, Chase HW, Bevan S. TRPM8 activation by menthol, icilin, and cold is differentially modulated by intracellular $\mathrm{pH}$. J Neurosci 2004; 24: 5364-9.

4 Andersson DA, Nash M, Bevan S. Modulation of the cold-activated channel TRPM8 by lysophospholipids and polyunsaturated fatty acids. J Neurosci 2007; 27: 3347-55.
5 Hilgemann DW, Feng S, Nasuhoglu C. The complex and intriguing lives of PIP2 with ion channels and transporters. Sci STKE 2001; 2001: re19.

6 Suh BC, Hille B. PIP2 is a necessary cofactor for ion channel function: how and why? Annu Rev Biophys 2008; 37: 175-95.

7 Nilius B, Owsianik G, Voets T. Transient receptor potential channels meet phosphoinositides. EMBO J 2008; 27: 2809-16.

8 Zakharian E, Cao C, Rohacs T. Gating of transient receptor potential melastatin 8 (TRPM8) channels activated by cold and chemical agonists in planar lipid bilayers. J Neurosci 2010; 30: 12526-34.

9 Kim AY, Tang Z, Liu Q, Patel KN, Maag D, Geng Y, et al. Pirt, a phosphoinositide-binding protein, functions as a regulatory subunit of TRPV1. Cell 2008; 133: 475-85.

10 Patel KN, Liu Q, Meeker S, Undem BJ, Dong X. Pirt, a TRPV1 modulator, is required for histamine-dependent and -independent itch. PLoS One 2011; 6: e20559.

11 Tang Z, Kim A, Masuch T, Park K, Weng H, Wetzel C, et al. Pirt functions as an endogenous regulator of TRPM8. Nat Commun 2013; 4: 2179.

12 Gao XF, Feng JF, Wang W, Xiang ZH, Liu XJ, Zhu C, et al. Pirt reduces bladder overactivity by inhibiting purinergic receptor $\mathrm{P} 2 \mathrm{X} 3$. Nat Commun 2015; 6: 7650.

13 Huang CL, Feng S, Hilgemann DW. Direct activation of inward rectifier potassium channels by PIP2 and its stabilization by Gbetagamma. Nature 1998; 391: 803-6.

14 Sui JL, Petit-Jacques J, Logothetis DE. Activation of the atrial KACh channel by the betagamma subunits of $\mathrm{G}$ proteins or intracellular $\mathrm{Na}^{+}$ ions depends on the presence of phosphatidylinositol phosphates. Proc Natl Acad Sci U S A 1998; 95: 1307-12.

15 Zhang H, He C, Yan X, Mirshahi T, Logothetis DE. Activation of inwardly rectifying $\mathrm{K}^{+}$channels by distinct Ptdlns(4,5)P2 interactions. Nat Cell Biol 1999; 1: 183-8.

16 Du X, Zhang H, Lopes C, Mirshahi T, Rohacs T, Logothetis DE. Characteristic interactions with phosphatidylinositol 4,5-bisphosphate determine regulation of kir channels by diverse modulators. J Biol Chem 2004; 279: 37271-81.

17 Ramsey IS, Delling M, Clapham DE. An introduction to TRP channels. Annu Rev Physiol 2006; 68: 619-47.

18 Hardie RC. Regulation of TRP channels via lipid second messengers. Annu Rev Physiol 2003; 65: 735-59.

19 Runnels LW, Yue L, Clapham DE. The TRPM7 channel is inactivated by PIP(2) hydrolysis. Nat Cell Biol 2002; 4: 329-36.

20 Liu D, Liman ER. Intracellular $\mathrm{Ca}^{2+}$ and the phospholipid PIP2 regulate the taste transduction ion channel TRPM5. Proc Natl Acad Sci U S A 2003; 100: 15160-5.

21 Chuang HH, Prescott ED, Kong H, Shields S, Jordt SE, Basbaum Al, et al. Bradykinin and nerve growth factor release the capsaicin receptor from Ptdlns(4,5)P2-mediated inhibition. Nature 2001; 411: 957-62.

22 Prescott ED, Julius D. A modular PIP2 binding site as a determinant of capsaicin receptor sensitivity. Science 2003; 300: 1284-8.

23 Stein AT, Ufret-Vincenty CA, Hua L, Santana LF, Gordon SE. Phosphoinositide 3-kinase binds to TRPV1 and mediates NGFstimulated TRPV1 trafficking to the plasma membrane. J Gen Physiol 2006; 128: 509-22.

24 Lukacs V, Thyagarajan B, Varnai P, Balla A, Balla T, Rohacs T. Dual regulation of TRPV1 by phosphoinositides. J Neurosci 2007; 27: 7070-80. 